\title{
Prevelance of Mesiodens in South Karnataka Population: A Retrospective Study
}

\author{
Henna P. S. ', George Babu ${ }^{2}$, Anusha Jayadevan ${ }^{3}$, Apoorva ${ }^{4}$
}

1. Dr. Henna P S, Postgraduate student, Dept.of Pedodontics and preventive dentistry, Coorg Institute of Dental Sciences, Virajpet.

2. Dr. George Babu, Reader, Dept.of Pedodontics and preventive dentistry, Coorg Institute of Dental Sciences, Virajpet.

3. Dr. Anusha Jayadevan, Postgraduate student, Dept. of Pedodontics and preventive dentistry, Coorg Institute of Dental Sciences, Virajpet.

4. Apoorva D, Undergraduate student, Dept. of Pedodontics and preventive dentistry, Coorg Institute of Dental Sciences, Virajpet.

\section{Corresponding author:}

Dr. Henna PS

Postgraduate student,

Department Of Pedodontics And Preventive Dentistry

Coorg Institute of Dental Sciences, Virajpet

Phone: 8861790512,8892304164

Email-hennasalim100@gmail.com

\begin{abstract}
Mesiodens is one of the most commonly seen supernumerary tooth in Indian population and they significantly affect primary dentition, mixed dentition as well as permanent dentition. Aim: To assess the prevelance of mesiodens in the South Karnataka population reporting to the department of Pedodontics in the duration of last 10 years. Design: In a 10 year retrospective study, a total of 40100 patient records up to the age of 12 years who visited our department between 01.01.2009 10 to 31.12.2018 were analysed. 11 cases of mesiodens were found and the selected patient records were evaluated for morphology, position, location, number, malocclusion, timing of the removal and the behavior assessment of the child along with the treatment plan used for the removal of mesiodens. Results: 11 subjects with mesiodens ( 8 males and 3 females) were detected, revealing male-female ratio of $2.6: 1$. The most common shape was conical and out of this 6 were impacted and 8 of them caused malocclusion, the orientation of the mesiodens and behavior of the child played a important role in treatment plan. Conclusions: The prevalence of mesiodens in South Karnataka paediatric population is $0.027 \%$ with slight male predilection and conical mesiodens being the commonest.
\end{abstract}

Key words: Mesiodens, Supernumerary teeth, Prevelance 


\section{Introduction}

Mesiodens is a specific type of supernumerary teeth that occurs in the midline of the arch in between the maxillary central incisors. ${ }^{1}$ They are seen most common among the types supernumerary teeth and is common in males.

They are also seen in various positions and orientations. ${ }^{1}$ In general they remain impacted and are commonly discovered during routine radiographic examination. ${ }^{2}$

There are several hypotheses which have been proposed to explain the occurrence of mesiodens. A combination of environmental and genetic factors have been proposed to explain the occurrence of mesiodens / supernumerary teeth ${ }^{5}$. The most popular theories include intrusion injuries to deciduous teeth, genetic predilection and hyperactivity of dental lamina ${ }^{3}$ and their etiology remains unclear.

The tooth morphology of mesiodens is often characterized by conical crown and a short root. Occasionally they also present as tuberculate or supplemental (tooth like). About $90 \%$ of the reported mesiodens are situated palatally 3 to the associated permanent incisors and rarely it is seen labially. Clinically it can present as single or multiple and can be inverted, vertical or horizontal in orientation.

Mesiodens results in variety of complications such as impaction of adjacent incisor, crowding, spacing, displacement and rotation of the teeth, occlusal interferences, caries, periodontal problem and esthetic concerns ${ }^{2}$ if it is intimately positioned to the permanent tooth bud it may alter the developing tooth bud, impede eruption, alter root development, and resorb roots of the existing incisors $^{3}$. Very rarely it is associated with complications like dentigereous cyst ${ }^{1}$. At times it can be associated with systemic disorders like cleidocranial dysplasia cleft palate and gardners syndrome ${ }^{4}$.

As a consequence of theses clinical implications, it is recommended that mesiodens be removed surgically. Early diagnosis and treatment of patients with mesiodens is important to prevent complications $^{5}$. The timing of removal of mesiodens after identifying clinically or radiographically depends up on the type of dentition (primary, mixed or permanent) and their co -operation ability.

A retrospective study was conducted using patient's records to understand the incidence of mesiodens in the pediatric patients reporting to the department and the timing of removal of mesiodens followed.

\section{Materials and Methods}

A retrospective prevalence study was planned. Patients record of 10 years retrospectively from 01.01.2009 10 to 31.12.2018 from the department OP records after obtaining permission from institutional review board was taken. Total of 11 patient records diagnosed with mesiodens was selected out of 40100 . The selected patient records were evaluated for the mention of morphology, position, location, number, timing of the removal of mesiodens and the behavior assessment of the child along with the information on sedation techniques used for the removal of mesiodens (chart 1).

\section{Results:}

The prevalence of mesiodens reported to the department in the 9 years duration was $0.027 \%$.

The age group observed was 7-12 years with male to female ratio of $2.6: 1$. The origin of the population with mesiodens was from Kerala, a neighboring state to Coorg. Out of the 11 mesiodens reported most of them were single mesiodens (8) (figure 1), and only in one case it was seen in both maxillary and mandibular arches. All the mesiodens reported were conical in shape out of these 6 were impacted while 8 were erupted (figure 2). 8 of them 


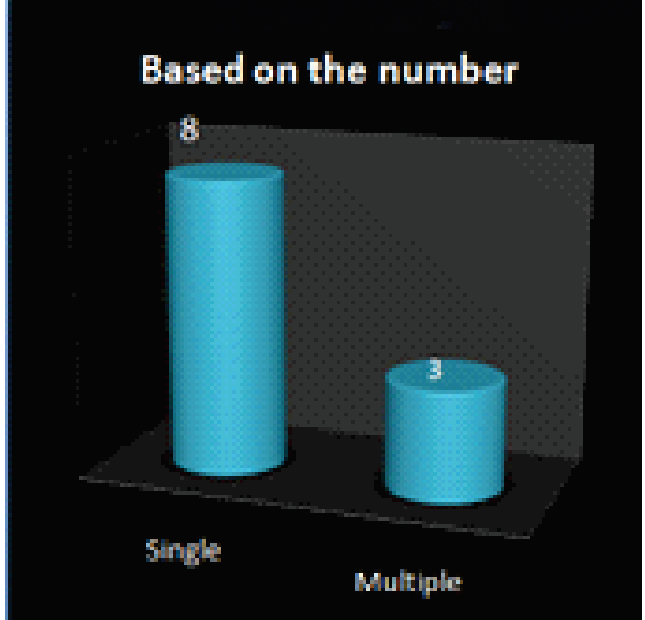

FIG. 1

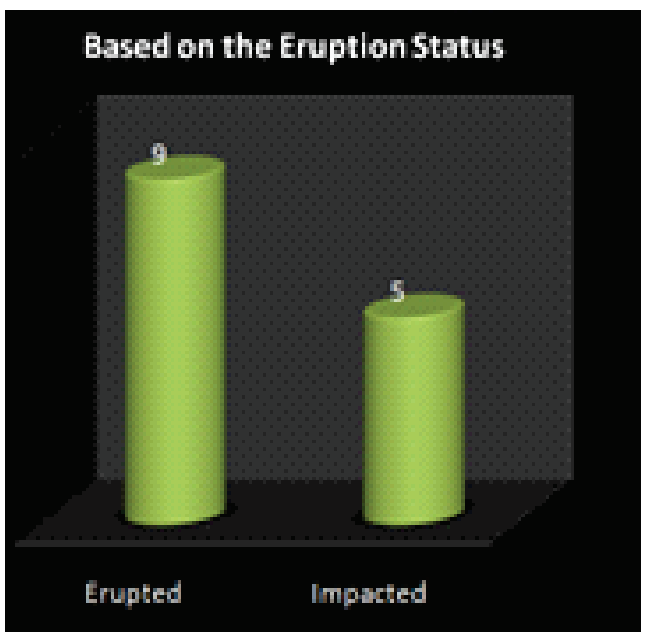

FIG. 2

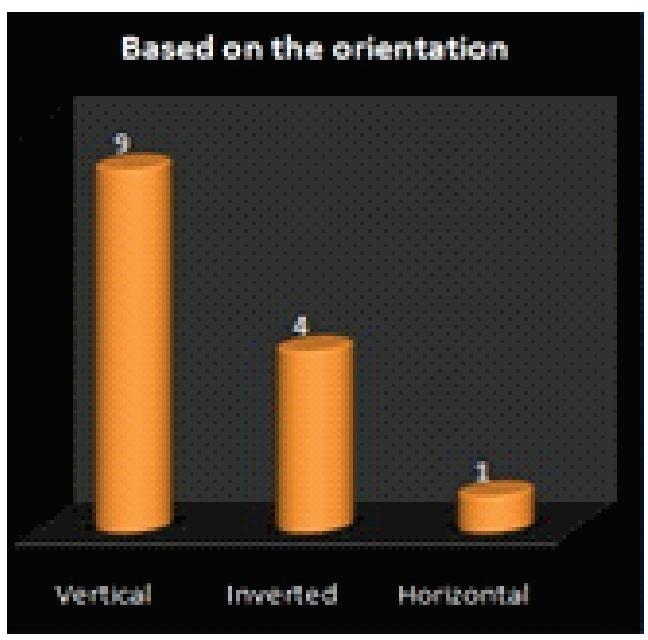

FIG. 3

had malocclusion due to presence of mesiodens and majority were vertically oriented (9) (figure 3).

The impacted mesiodens were removed under GA or conscious sedation. GA was done on the children who were assessed uncooperative (frankel behavior rating 1 and 2) and conscious sedation was done in an otherwise co -operative child. The erupted mesiodens was removed without sedation, but using chair side distraction techniques.

\section{Discussion and Review Of Literature}

Supernumerary teeth are developmental anomalies of number which are observed during regular dental checkup. Various studies have described the prevalence and gender predilection in different populations. (chart 2). According to these studies the prevalence of mesiodens is $0.45 \%$ in Caucasians, $1.43 \%$ in Norwegians, $0.3 \%$ Turkish population and $0.4 \%$ in Finnish population. 6 in Coorg population it was found to be $0.02 \%$. Which is low compared to other populations reported.

Mesiodens are predicted more in boys than in girls. According to sung-suk lee the ratio in Korean population was $3.7: 1$ which seems to be slightly higher but most of the studies showed the ratio between $1.8: 1$ to $2.05: 1$. In this population male predilection was observed with ratio of 2.6:1

The shape of mesiodens vary from conical to tubercle to supplemental or odontome. Conical is the most common type seen according to Krishnan Ramesh et al (79.74\%) 7 Yuh Yun Kim et al 8(96.1\%), Santhosh Patil et al(80.1\%) . mesiodens morphology observed were conical in the present study (\%).

The orientation of the mesiodens observed had an increased incidence of vertical type (62.5\%) . This was in accordance with the study conducted by Sungsuk Lee $9(62.3 \%)$ and it was higher compared to the studies by Liu et al, Gunduz et al, Roy Chandani et al (46\%,55.2\%, 31.25\% respectively). The inverted type was $31.25 \%$ and horizontal was $6.25 \%$.

In pediatric patient the behavior has significant role in treatment plan. From the data we have noticed that the extraction of the teeth and the timing 


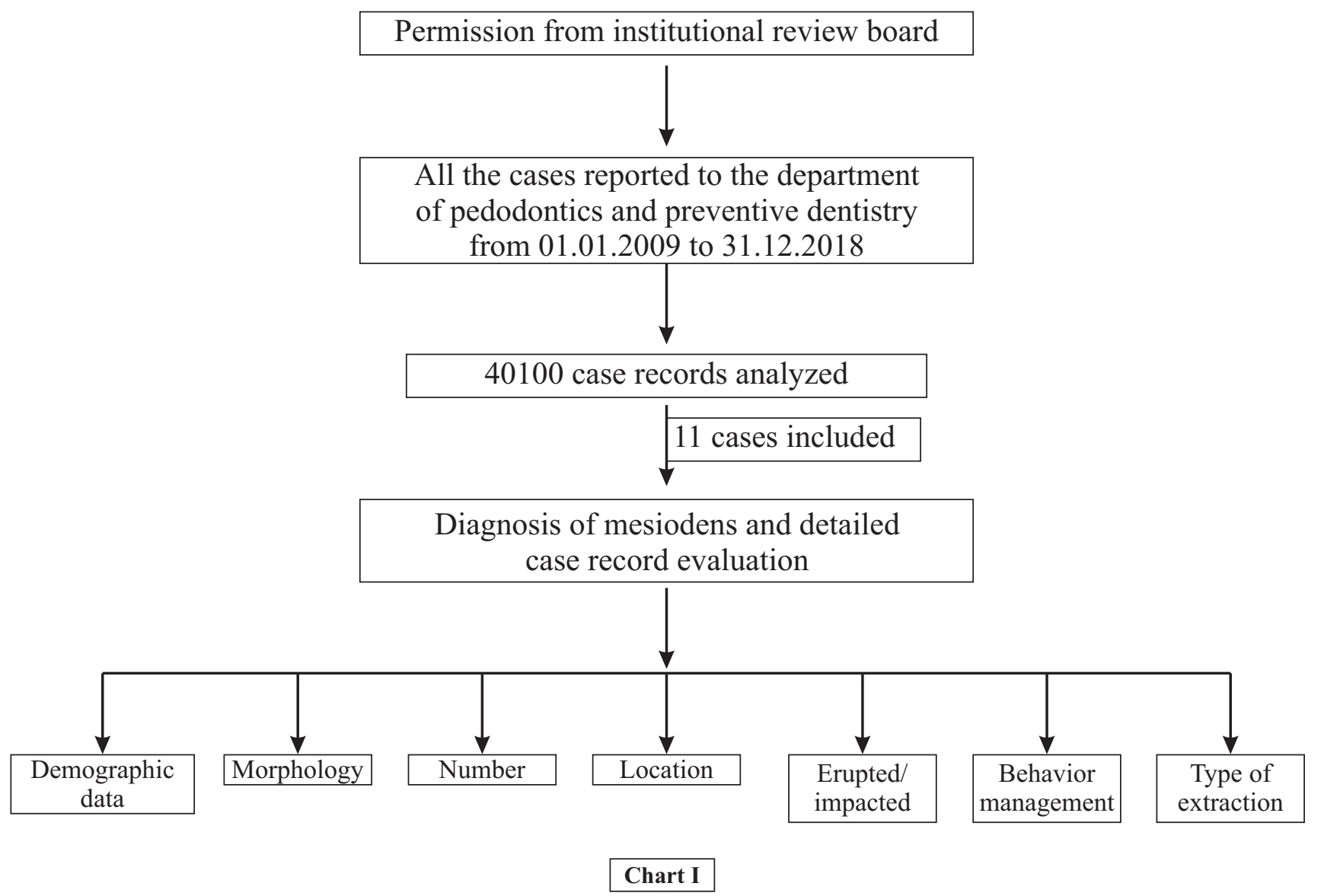

was decided according to the age and behavior of the child. Four patients underwent extraction under LA chair side since they had a positive behavior. A positive behavior patient who was slightly anxious was treated under audio analgesia and conscious sedation .children who were definitely negative in behavior and had impacted mesiodens were treated under general anesthesia

\begin{tabular}{l|l|l|l|} 
& $\begin{array}{l}\text { International } \\
\text { (South Korea) }\end{array}$ & North India & $\begin{array}{c}\text { Coorg and } \\
\text { Kerala }\end{array}$ \\
\hline Year & $2009-2013$ & $2008-2012$ & $2009-2019$ \\
\hline Male to Female ratio & $3.7: 1$ & $1.8: 1$ & $2.6: 1$ \\
\hline Single mesiodens & $70.1 \%$ & $89.7 \%$ & $72.7 \%$ \\
\hline Multiple mesiodens & $29.6 \%$ & $5 \%$ & $27.2 \%$ \\
\hline Impacted & & $62.9 \%$ & $37.5 \%$ \\
\hline Erupted & & $22.5 \%$ & $62.5 \%$ \\
\hline Vertical & $62.4 \%$ & $59.6 \%$ & $62.5 \%$ \\
\hline Inverted & $26.0 \%$ & $25.8 \%$ & $31.25 \%$ \\
\hline horizontal & $11.6 \%$ & $14.6 \%$ & $6.25 \%$ \\
\hline \hline
\end{tabular}

TABLE - 1
Early diagnosis is crucial in the management of mesiodens in this study it has been found that extraction of mesiodens in early mixed dentition period showed less complications in the further follow up visits. But in patients in the late mixed dentition period with the mesiodens were identified to have more deleterious consequences like malpositioning of incisors, delayed eruption, crowding and decreased oral hygiene. This was in accordance with the study by Russel et al 6 in which he recommended extraction of mesiodens in the early mixed dentition stage for better positioning of teeth and thereby reducing the need for orthodontic treatment. However the periodic recall and checkup plays a vital role in the prognosis.

\section{Conclusion}

Pediatric dentist should consider early diagnosis of mesiodens, intervention and prevent many possible complications which can cause potential 
harm to the developing occlusion in a child patient, which can be difficult to intervene or may require aggressive treatment at later stage.

The factors to be considered before intervention along with the position and location of tooth are; cooperation of the child -parent and appropriate behavior modification techniques based on not only the co-operation level but also depending on type of procedure required.

\section{References}

1. Cone beam computed tomography review and classification of mesiodens: Report of a case in the nasal fossa and nasal septum,Albert A, Mupparapu M, Quintessence Int. 2018; 49(5):413-417.

2. Cone-beam computed tomography exploration and surgical management of palatal, inverted, and impacted mesiodens, Omami M, Chokri A, Hentati H, and Selmi J, Contemp Clin Dent. 2015 Sep; 6(Suppl 1): S289-93.

3. Characteristics of Mesiodens and Its Related Complications, Nam OH, Lee HS, Kim MS, Yun KH, Bang JB, Choi SC, Pediatr Dent. 2015; 37(7):E105-9

4. Effects of mesiodens on adjacent permanent teeth: A Retrospective study in Korean children based on cone-beam Computed tomography,
Kim Y, Jeong T, Kim J,Shin J, Shin K, Int J Paediatr Dent .2018,28(2);161-69

5. Mesiodens: A clinical and radiographic study of 82 teeth in 55 children below 14 years, Ramesh K, Venkataraghavan K, Shiji K and Maya R, J Pharm Bioallied Sci. 2013 Jun; 5(Suppl 1): S60-S62

6. Frequency of mesiodens in the pediatric population in North India: A radiographic study Patil Ss, Pachori Y, Sumita K,Khandelwal S, Likhyani L, Sneha M, J Clin Exp Dent. 2013 Dec; 5(5): e223-e226.

7. Fusion of maxillary central incisors with mesiodens, Madhuram K, V Kumar, Leburu A, Nadeem J, J Oral Maxillofac Pathol. 2018 Jan; 22(Suppl 1): S131-S134.

8. Prevalence and characteristics of supernumerary teeth in a non-syndromic South Indian Pediatric population, Rajesh A, Tegginmani V, Battepati P, Tavargeri A, Patil S, Vijay T, Garima J, J Indian Soc Pedod Prev Den, $2014: 32(1) ; 9-12$.

9. A comparative analysis of patients with mesiodenses: a clinical and radiological study. S,Kim S , Oh J, You J, Jeong K, Kim Y,Lee S, Lee N, J Korean Assoc Oral Maxillofac Surg. 2015 Aug; 41(4): 190-193. 